\title{
Sexual selection and mate choice
}

\section{Malte Andersson ${ }^{1}$ and Leigh W. Simmons ${ }^{2}$}

\author{
${ }^{1}$ Department of Zoology, University of Gothenburg, SE 40530 Gothenburg, Sweden \\ ${ }^{2}$ Centre for Evolutionary Biology, School of Animal Biology (M092), The University of Western Australia, Crawley 6009, WA, \\ Australia
}

The past two decades have seen extensive growth of sexual selection research. Theoretical and empirical work has clarified many components of pre- and postcopulatory sexual selection, such as aggressive competition, mate choice, sperm utilization and sexual conflict. Genetic mechanisms of mate choice evolution have been less amenable to empirical testing, but molecular genetic analyses can now be used for incisive experimentation. Here, we highlight some of the currently debated areas in pre- and postcopulatory sexual selection. We identify where new techniques can help estimate the relative roles of the various selection mechanisms that might work together in the evolution of mating preferences and attractive traits, and in sperm-egg interactions.

\section{Introduction}

Twenty years ago, when sexual selection was reviewed in the first issue of TREE [1], it was a rapidly growing field. Darwin's idea of female preferences for male ornaments was still controversial, although his theory had received new support from two directions. First, empirical studies showed that male ornaments are favoured by female choice in some fishes and birds [1]. Second, a major problem left open by Darwin, the reasons why females prefer ornamented males, was clarified when genetic models [2,3] verified the logical coherence of Fisherian self-reinforcing coevolution of male ornaments and female preferences (Box 1).

These results posed as many interesting questions as they answered, and inspired much new research. In the two decades since 1986, sexual selection theory has been corroborated [4] and enriched with exciting new ideas and discoveries, some of which we highlight here. We also point to new possibilities for testing genetic mechanisms of sexual selection in the era of functional genomics. Genetic analyses of sexual selection by mate choice have worked so far on a mainly top-down basis, inferring genetic causes from phenotypic patterns, based on fewlocus genetics or quantitative genetics theory [5]. These remain excellent tools for the analysis of preferencedisplay coevolution and for other purposes in sexual selection research. However, quantitative trait locus (QTL) identification and sequencing combined with functional genomics now provide the opportunity for bottom-up approaches, based on the precise

Corresponding author: Andersson, M. (malte.andersson@zool.gu.se)

Available online 3 April 2006 characterization of genes and their effects, from DNA sequences via protein to phenotypic expression at the level of the individual, with possible consequences at the population level and above.

\section{Evolution of mate choice}

Although mate choice occurs in males and females [4], for convenience we refer here to female choice of male traits. As experimental evidence accumulated, mate choice became widely recognized, but the genetic mechanisms underlying its evolution remain the subject of debate (Box 1). Showing how mating preferences evolve genetically is harder than showing that they exist, and the problem is aggravated by the possibility that several mechanisms co-occur (Box 1). Moreover, conflicts between the sexes can add further selection pressures on preference and the preferred trait [6,7]. Opinions differ over the relationships between Fisherian and genetic indicator mechanisms of mate choice (e.g. [5,8-13]). Given that there are qualitative differences between them, we think there are good reasons to keep the distinction clear $[9,11,12]$.

Costs of mate choice, such as the loss of energy and time, might prevent Fisherian self-reinforcing coevolution of the trait and the preference for it, but need not do so; the outcomes of models depend on the details of several assumptions, for instance about sex linkage [10] (reviewed in [5]). In spite of being theoretically plausible, the Fisherian genetic mechanism has been difficult to demonstrate empirically. There is corroborating phenotypic evidence (e.g. $[14,15])$, and this is also the case for indicator mechanisms (e.g. reviewed in $[5,11,16]$ ). These and other mechanisms (Box 1) are compatible and might co-occur (e.g. $[4,12]$ ), and thus a challenging task is to gauge their relative roles. Estimating the effects of the Fisherian sexy son mechanism might help us to decide whether it explains why male ornaments and displays are often extreme, and differ more than do other traits between closely related species, apparently evolving rapidly and perhaps being involved in speciation $[2,17,18]$. These questions have been with us since Charles Darwin's time and have yet to be answered satisfactorily.

\section{Testing the evolution of trait and preference}

Molecular genetic and genomic tools enable the detailed characterization of genes and their effects (e.g. reviewed in [19-21]). Combined with selection experiments that quantify genetic evolution over generations, they offer tools for detailed genetic analyses and tests of whether 


\section{Box 1. Mechanisms of mate choice evolution}

Several mechanisms have been put forward to explain mate choice: (i) Direct phenotypic effects. Female preference for a male ornament can evolve as a result of direct phenotypic benefits if the ornament reflects the ability of the male to provide material advantages, such as a high-quality territory, nutrition, parental care or protection. There is considerable empirical support for this mechanism [76]. Female choice might also evolve as a result of resistance to direct costs imposed by males $[7,38]$.

(ii) Sensory bias. Female preference favouring a male ornament can initially evolve under natural selection for other reasons, for instance in the context of foraging or predator avoidance (e.g. $[22,77])$. Males evolving traits that exploit this bias then become favoured by mate choice. There is increasing phenotypic evidence that some male ornaments initially evolved through female sensory biases $[22,77,78]$, but the evolution of female sensory bias itself requires more testing [12].

(iii) Fisherian sexy sons. If there are genetic components to variance in female preference and male trait, a female choosing a male with a large trait bears daughters and sons that can both carry alleles for a large trait, and for the preference for it. This genetic coupling might lead to self-reinforcing coevolution between trait and preference $[2,3,5,10,12,17]$. Direct critical testing of this mechanism is difficult, but molecular genetics offers new possibilities (see main text).

(iv) Indicator mechanisms ('good genes' or 'handicap mechanisms') suggest that attractive male traits reflect broad genetic quality. Inherent in such mechanisms is the maintenance of genetic variation, the 'paradox of the lek', and parasite- and pathogenmediated mechanisms have been suggested as potential solutions (Box $4[79,80])$. In addition, other advantageous genes and relative freedom from deleterious mutations might lead to high male condition and expression of sex traits (e.g. [11,81-83]). Female preference for such traits can provide genetic benefits to those of her offspring that inherit favourable alleles from their father (e.g. reviewed in $[5,11,13,84]$, but see [85]). The resolution of the lek paradox remains a crucial area for sexual selection research.

(v) Genetic compatibility mechanisms. As well as additive genetic benefits reflected by indicator traits, there might be non-additive benefits from choosing a mate with alleles that complement the genome of the chooser $[37,65-68,84]$. Examples have been found for instance in major histocompatibility complex genes, and compatibility advantages might be one adaptive reason for multiple mating by females (see main text).

The evolution of mate choice is based either on direct selection of a preference that gives a fitness advantage (mechanisms i-ii) or on indirect selection of a preference as it becomes genetically correlated with directly selected traits (mechanisms iii,iv) [4,5,10,12]. In addition, rather than favouring any particular display trait, mate choice might evolve because it conveys non-additive genetic benefits (mechanism v) $[65,66]$. These mechanisms are mutually compatible and can occur together, rendering the evolution of mating preferences a multiple-causation problem, and calling for estimation of the relative roles of individual mechanisms [4]. Several diagnostic differences among the mechanisms suggest ways in which they can be tested by quantitative genetic analyses $[5,12]$.

mating preferences and preferred traits evolve as predicted by sensory bias, Fisherian, indicator, or other mechanisms.

There is evidence for sensory bias in an increasing number of species, where phylogenetic analyses have shown that female preference for a male display trait is also present in other related species lacking the male trait (e.g. [22-24]). In such cases, it seems likely that the female preference arose first for other reasons, such as attraction to food (which needs to be tested [12]), hereafter the male trait evolved, favoured by the pre-existing female sensory bias [22].

The Fisherian mechanism is, in essence, based only on genes that influence mating preferences and preferred traits, and it can lead to their rapid coevolution if the most preferred males are polygynous (e.g. [2,5,10,17]). Genetic indicator mechanisms, however, depend on overall quality and hence on much or most of the functional genome; they can also work in monogamous systems where all males obtain a mate $[11,12]$. These differences suggest ways in which the two processes can be distinguished and their relative importance estimated.

A prediction from the Fisherian mechanism is that the expression of trait and corresponding preference are positively correlated, and this has been found in several species. However, such a correlation could also arise from other mechanisms of male-female coevolution $[4,8]$ (Box 1).

\section{Bottom-up genetic testing}

One possible approach to testing is to identify, sequence, and characterize in functional detail a gene locus with several alleles that influence the expression of an attractive male trait, and to do likewise for the corresponding female preference. Although there are many difficulties, a combination of molecular genetic tools can make such analyses feasible in suitable model organisms (Box 2). Chromosomal regions hosting genetic variation with substantial influence on the expression of trait or preference can be identified by QTL analysis $[19,25]$, followed by nucleotide characterization of the alleles at the loci. Techniques such as DNA microarrays, which can detect expression-level polymorphism, are useful in gene hunting for variable loci that influence the phenotypic traits of interest (e.g. $[19,21,26]$ ), as are candidate gene approaches $[20,26]$.

If several alleles can be sequenced that encode for different degrees of expression of the trait, and likewise for the preference, genetic tests of the Fisherian process might be possible, for example by finding out whether alleles for trait and preference coevolve as predicted. Selective breeding over several generations from those males with greatest (or smallest) trait expression could show whether and how preference alleles coevolve with trait alleles, and vice versa. In combination with microsatellite analysis of parentage, it might also be possible to measure mating and fertilization success, and to quantify over several generations the Fisherian sexy sons advantage in relation to male trait size.

How can the genetic effects of Fisherian and indicator processes be distinguished in such experiments? In species where only males express the attractive trait and do not provide parental care or other phenotypic resources, daughters can be tested for genetic viability effects that are correlated with the indicator trait of their father, to find out whether it reflects a viability advantage, which is not predicted by the Fisherian mechanism. Maternal half-sib designs [27] can be particularly useful. Experimental designs could also be used where Fisherian mating advantages are prevented, enabling a male to mate only with a single female. There are other possibilities; for example, if an attractive male trait is also expressed to some extent in females, although at a cost, the added effect of intralocus sexual conflict [7] might 


\section{Box 2. Model systems in sexual selection}

The properties desirable in a model system for analysis of sexual selection and mate choice include:

- A model species should be amenable to comprehensive study in the natural environment, as a major goal is to understand how sexual (and other) selection works in the wild, where species evolve and acquire their characteristics. Genetic and other experimental approaches, many of which are feasible only in the laboratory, can then be interpreted against a solid background of ecology, selection pressures and adaptations of the species in its natural evolutionary context.

- A model system should involve conspicuous sexual dimorphism in structure or behaviour, as these are common correlates of strong sexual selection.

- Model organisms should have small body size and short generation time, as these are most suitable for experimental analysis of selection and its evolutionary consequences over a sufficient number of generations.

- Ideally the species, or a close phylogenetic relative, should already be genetically well known, to facilitate crucial microevolutionary tests of sexual selection.

These properties are available in several insects and fishes, such as Drosophila fruit flies (Figure la, reproduced with permission from Tracey Chapman), Cyrtodiopsis stalk-eyed flies (Figure lb, reproduced with permission from Sam Cotton), threespined stickleback Gasterosteus aculeatus (Figure Ic, reproduced with permission from Massimo Lorenzoni) and guppy Poecilia reticulata (Figure Id, reproduced with permission from Ann Houde). These are all subjects of incisive research programs, as they make excellent models for combined genetic and phenotypic analyses of evolution by sexual and other forms of natural selection. For example, experimental studies of life-history evolution and tradeoffs between sexual selection and predation have been done in wild populations of guppies (reviewed in [34,35]). Reproducing readily and with short generation time in captivity, the guppy has also proved ideal for microevolutionary analyses in the laboratory. Recently, it was shown that the orange colour spots in males preferred by females might be colour mimics of important fruit food for the guppy, a possible example of sensory bias (Box 1) [23]. Orange coloration also correlates with postcopulatory processes that bias paternity towards preferred males [32]. When a genome database becomes available for the guppy (which should be high priority), important steps are likely to be taken towards a deeper understanding of the sexual and other selection mechanisms that are driving genetic microevolution in nature.
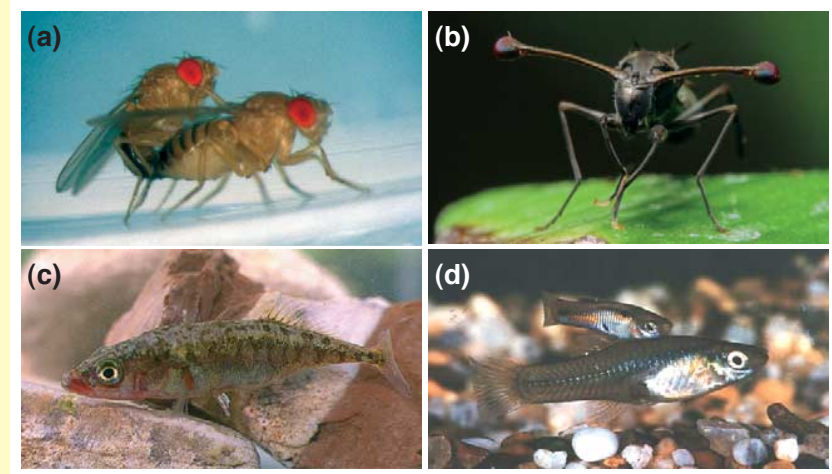

Figure I.

be estimated by measuring the fitness effect of the trait in females as well as in males.

Prospects for success in such tests seem best in model species (Box 2), where much genetic information is already available (also see [28]). For example, in Drosophila, QTL analyses have identified loci that cause variation in courtship traits, such as male song and female mate recognition $[29,30]$. Ecologically interesting phylogenetic relatives of genomic model species could also be used by taking advantage of a candidate gene approach, based on knowledge of the model species [19,20], because gene function is sometimes phylogenetically conserved. For instance, information from the genome databases of pufferfish Tetraodon nigroviridis and zebrafish Danio rerio could be useful in searching for trait and/or preference loci in two frequent targets of sexual and natural selection analyses, the guppy (e.g. [23,31-35], Box 2) and the threespined stickleback (e.g. [24,27]).

\section{Mate choice and sexual conflict}

The importance of conflict between the sexes is becoming increasingly clear through a flood of exciting recent research [7]. Early insights of Williams [36], Trivers [37] and, especially Parker [6], have recently received much support, and it now appears that sexual conflict between males and females is the rule rather than the exception, sometimes leading to sexually antagonistic coevolution (e.g. [38,39]).

In terms of mate-choice evolution (Box 1), sexual conflict can impose direct selection on the female preference that leads to increased female resistance to the male trait, because its consequences are negative for females [40]. Many aspects of sexually antagonistic coevolution, such as its co-occurrence with indirect selection on mate choice (Box 1), are debated (e.g. $[7,9,13,38,39,41])$. Combinations of genetic and phenotypic approaches might help resolve these issues (reviewed in [7]).

\section{Mate choice or paternity choice?}

One of the main advances in sexual selection theory since Darwin was the realization by Parker [42] that selection can continue after copulation. If a female mates with more than one male, there will be competition among sperm of different males to fertilize available ova. In the 35 years since Parker's paper, sperm competition has become a major branch of sexual selection research [43].

Initially, the evolution of traits such as male genital morphology and ejaculate size were attributed to sexual selection for male engagement in sperm competition. Traits such as mating plugs, seminal fluid peptides that influence female reproductive physiology, and postcopulatory mate guarding, were interpreted as male adaptations for the avoidance of sperm competition. Multiple mating by females is a prerequisite for sperm competition, yet it is only in the past decade that postcopulatory sexual selection has been considered from the female perspective [44,45]. Bateman [46] suggested that female fitness is maximised by mating with one or a few males. However, this view is now shifting rapidly to one in which females are thought to often obtain direct phenotypic and indirect genetic benefits from multiple mating [47]. Whereas sperm competition is viewed as the postcopulatory equivalent of male contest competition, the corresponding equivalent of female choice is referred to as cryptic female choice, because we are unable to observe directly female 


\section{Box 3. Problems in demonstrating cryptic female choice}

Females could bias paternity toward preferred mates by a range of potential mechanisms [45], the easiest of which to demonstrate occur during copulation. Females of some species expel sperm following copulations with some males yet accept sperm from others, biasing paternity (e.g. $[43,86])$. More often, only the numbers of sperm found in storage after single matings, or patterns of paternity following multiple matings, can be determined. The sperm stored might reflect biases of cryptic female choice imposed during the travel of sperm within her reproductive tract $[43,45]$. The numbers of sperm might also reflect adaptive male responses to the reproductive value of the female (i.e. cryptic male choice [87]). As regards relative paternity among several males, it is equally difficult to distinguish between male and female processes $[43,88]$ and to show unequivocally whether paternity reflects sexual selection at the gametic level.

An increasing number of studies have found patterns of paternity in offspring that can be interpreted as selective fertilization by genetically superior (e.g. $[32,89])$ or compatible sperm haplotypes, either among sperm from different [90] or single males [91]. Such studies typically assign paternity using DNA or morphological markers scored from newly born or adult offspring. The patterns of paternity could reflect the outcome of interactions between sperm and egg, male success in sperm competition, or preferential use of particular sperm haplotypes by females. However, this interpretation can be confounded by viability consequences of genetic incompatibilities, or by intrinsic sire effects on offspring viability [47]. To demonstrate unequivocally that patterns of paternity reflect sperm competition or cryptic female choice, paternity should be determined at a very early stage, before the onset of embryo mortality. Nevertheless, recent work on free-spawning invertebrates provides good evidence that sperm-egg interactions can generate selective fertilization [47].

preferences for sperm from particular males. Rather, they manifest as biases in paternity (Box 3 ).

Thus, mate choice by females can be viewed as the first step in the process of paternity choice. The incorporation of female perspectives into postcopulatory sexual selection has generated theoretical models for the evolution of multiple mating by females that in some ways parallel the mechanisms of mate choice evolution in Box 1. For example, the 'sexy-sperm' hypothesis parallels the Fisherian sexy sons mechanism in proposing that multiply mating females will have sons with high fertilization success, because they inherit traits that caused high fertilization success for their fathers. These traits can include genital morphology, number and/or quality of sperm, or seminal fluid composition [48].

Similar to Fisherian sexy sons, the evolutionary potential of the sexy-sperm mechanism depends on the genetic architecture of traits that contribute to fertilization success [49]. Nevertheless, it has been argued that such a Fisherian mechanism might underlie the rapid and divergent evolution of male genital morphology, if females are more likely to store and use sperm from males able to provide the appropriate genital stimulation during copulation [50]. Under this scenario, male genitalia, traditionally viewed as primary sexual traits, can become postcopulatory equivalents of the peacock's train, questioning the utility of a distinction between primary and secondary sexual traits. There is increasing experimental support for such a notion [51,52].

The 'good-sperm' hypothesis suggests that the ability of a male to gain high fertilization success is correlated with his underlying genetic quality, so that males successful in sperm competition sire offspring with generally high viability [53]. The good-sperm hypothesis in this respect parallels indicator mechanisms for the evolution of male sexual ornaments. Male sexual ornaments subject to precopulatory sexual selection are often condition-dependent indicators, developing in proportion to male condition $[11,16]$. Perhaps directional postcopulatory sexual selection might likewise favour condition-dependent traits that influence paternity and thereby provide indirect genetic benefits for offspring (Box 4). There are few tests of this 'good-sperm' hypothesis, but the males of bulb mites [54] and dung flies [55] that are most successful in sperm competition sire offspring of higher fitness, and quantitative genetic studies of traits thought to be important in sperm competition suggest condition dependence in a beetle species [56].

\section{Interacting pre- and postcopulatory choice}

Although theoretically possible, there is currently little evidence that sperm competition and/or cryptic female choice can amplify precopulatory mate choice. Suggestive evidence comes from studies of guppies in which males with more orange body colouration, a trait subject to precopulatory female choice, have a paternity advantage following artificial insemination of females with equal numbers of sperm from two competing males [32]. Given that females had not met the sperm donors in these experiments, the most parsimonious explanation seems to be that males of intrinsically high quality have attractive sexual traits and also produce highly competitive sperm

\section{Box 4. Male germ line control: an opportunity for postcopulatory female choice?}

The immunocompetence handicap hypothesis $(\mathrm{ICHH})$ proposes that elaboration of secondary sexual traits under sexual selection, and success in sperm competition, have a common denominator in a male tradeoff with investment in immune function [79,92]. Immune responses to pathogens and parasites are suggested to be costly, so that individuals subject to high levels of infection are likely to have reduced resources available for secondary sexual display. The ICHH is thus an indicator mechanism of sexual selection, and proposes that females can obtain heritable resistance to disease for their offspring from those males who can afford to invest in large sexual displays. A postcopulatory extension of the $\mathrm{ICHH}$ suggests that immune defenses also compromise the ability of a male to produce high-quality ejaculates.

This hypothesis was originally formulated for vertebrates and assumed that the immunosuppressive properties of testosterone are required to counter attack by the diploid immune system of the male on his haploid sperm cells [92]. Similar to the precopulatory version of the $\mathrm{ICHH}$, the hypothesis can be generalised if we assume a resource allocation tradeoff between sperm production and immune function [93]. Males that are forced to fight infection by upregulating immune function pay a cost of reduced sperm quality [94]. Mating with multiple males and enabling sperm competition to filter the most successful, females might therefore produce offspring with the resistance to disease that enabled their fathers to produce sperm of superior quality. There is increasing evidence that males face a phenotypic tradeoff between investment in secondary sexual traits and immune function [95-97]. A recent quantitative genetic analysis in crickets provides evidence for a genetic tradeoff between sperm quality and immune function, as required by the postcopulatory extension of the ICHH [98]. 
(but see Box 3). Later behavioural experiments found that females accept more sperm from attractive males [33]. Both sperm competition and cryptic female choice might amplify precopulatory sexual selection in guppies. Measurement of the relative sexual selection intensities during pre- and postcopulatry phases of paternity choice seems a worthwhile endeavour, yet has rarely been attempted $[57,58]$.

Molecular genetic studies of parentage have revolutionised our views of avian mating systems, and multiple mating by avian females is known to result in widespread and highly variable rates of extra-pair paternity (EPP) [59]. In several species, EPP is most common in broods of females whose social mate is relatively unattractive and/ or of lower social status in precopulatory sexual selection $[59,60]$. If females engage in extra-pair copulations to trade up from their social mate and maximize offspring fitness, we might expect pre- and postcopulatory mate choice to work synergistically in favouring the evolution of male sexual ornaments. Comparative evidence suggests that sexual dichromatism correlates with rates of EPP across bird species, particularly with respect to structural colours used in sexual display [61]. But this relationship can also be explained by females timing their copulation behaviour to ensure that offspring are sired by extra-pair males. Thus, patterns of EPP might reflect precopulatory choice of extra-pair males rather than postcopulatory mate choice. An exciting new avenue for research on EPP in birds would be to explore correlations between male display traits and the fertilization capacity of their sperm in controlled experiments, to determine the relative importance of extra-pair copulations compared with postcopulatory processes in determining EPP. Artificial insemination techniques offer a promising approach.

Postcopulatory sexual selection can attenuate precopulatory sexual selection. It is now recognized that, counter to Bateman's principle [46], the reproductive success of a male is sometimes limited by the number of sperm that he can produce. Males who are successful in precopulatory sexual selection can have higher costs of sperm production and become sperm depleted, so that female fertility is sperm limited, and successful males can suffer reduced competitive fertilization success [62-64]. Moreover, one of the putative genetic benefits of postcopulatory female choice is the avoidance of genetic incompatibility between maternal and paternal haplotypes, so that the preferred sire of a female depends on her own genotype $[65,66]$. Thus, although attractive males might gain more copulations, their paternity can be reduced among females with whom their genes are incompatible. As a result, the strength of sexual selection on male ornamental traits can be reduced [67]. Integrating the effects of precopulatory female choice of males carrying good genes with postcopulatory choice of compatible genes is a new challenge in mate choice evolution [68].

\section{Postcopulatory sexual conflict}

Despite an early focus on male perspectives in sperm competition, Parker [42] recognized that females are not inert environments in which male adaptations to sperm competition arise. Traits such as male genital morphology, or accessory gland products that enhance male fitness in sperm competition, can impose costs on female fitness [69] and generate antagonistic coevolution between the sexes (e.g. [7,38,39]). Molecular genetic and genomic tools are now being used to clarify the proximate mechanisms of potentially strong postcopulatory sexual selection and conflict, for example by identifying gene loci and proteins of Drosophila seminal fluids. These proteins induce changes in the female that are beneficial to the male, such as increased egg laying and decreased receptivity to other males. Some of the substances are, however, harmful to and reduce life span in females, and so are generating selection via sexual conflict (reviewed in [7,70]). Nonetheless, such harmful male adaptations might also evolve via a Fisherian sexy sons process, if the net fitness benefit to the female from producing harmful sons outweigh the costs of harm to her (e.g. [41]).

Many male and female reproductive proteins involved in sperm-egg recognition and fertilization evolve rapidly, and differ strongly between closely related taxa (reviewed in $[7,18,71])$. For example, among 987 genes expressed at different times during mouse spermatogenesis, protein evolution was fastest for testis-specific genes expressed during late stages of sperm maturation and involved in sperm-egg interactions [72]. Although the mechanisms are not yet known, many of these genes show evidence of positive selection [72], as expected if there is sexual selection leading to rapid coevolution of male and female reproductive proteins $[7,72]$. Genetically engineered lines of Drosophila enable the estimation of the relative strengths of selection via antagonistic coevolution and indirect genetic benefits to females through their sons [73-75]. These studies illustrate how combinations of behavioural, biochemical and genetical experiments can produce further insights into sexual selection and conflict and their potential roles in speciation $[18,40]$.

\section{Conclusions}

Twenty years on we no longer think of sexual selection simply acting on male sexual ornaments. Traits as diverse as behavioural and structural displays, genital morphology and reproductive proteins can be subject to a multitude of selection pressures imposed by mate choice. The genetic mechanisms of pre- and postcopulatory sexual selection are still far from being fully understood, but molecular genetic and genomic tools enable their detailed experimental testing. This goal is likely to be achieved first in model organisms with sequenced genomes, or in some of their phylogenetic relatives. Progress will not be quick and easy, but we would not be surprised if the 40th anniversary issue of TREE is able to review detailed genetic clarification of Fisherian, indicator, mate compatibility, sperm competition, cryptic female choice, sexual conflict and other mechanisms of mate choice evolution. We thus look forward to the future of sexual selection with much curiosity and expectation.

\section{Acknowledgements}

We thank R. Marshall and the referees for constructive comments on the article, and the Swedish Research Council, the Swedish Foundation for 
International Cooperation in Research and Higher Education (M.A.) and the Australian Research Council (L.W.S.) for financial support.

\section{References}

1 Majerus, M.E.N. (1986) The genetics and evolution of female choice. Trends Ecol. Evol. 1, 1-7

2 Lande, R. (1981) Models of speciation by sexual selection on polygenic traits. Proc. Natl. Acad. Sci. U. S. A. 78, 3721-3725

3 Kirkpatrick, M. (1982) Sexual selection and the evolution of female choice. Evolution 36, 1-12

4 Andersson, M. (1994) Sexual Selection, Princeton University Press

5 Mead, L.S. and Arnold, S.J. (2004) Quantitative genetic models of sexual selection. Trends Ecol. Evol. 19, 264-271

6 Parker, G.A. (1979) Sexual selection and sexual conflict. In Sexual Selection and Reproductive Competition in Insects (Blum, M.S. and Blum, N.A., eds), pp. 123-166, Academic Press

7 Arnqvist, G. and Rowe, L. (2005) Sexual Conflict, Princeton University Press

8 Kokko, H. et al. (2002) The sexual selection continuum. Proc. R. Soc. B $269,1331-1340$

9 Cameron, E. et al. (2003) Sexual conflict and indirect benefits. J. Evol. Biol. 16, 1055-1060

10 Kirkpatrick, M. and Hall, D.W. (2004) Sexual selection and sex linkage. Evolution 58, 683-691

11 Andersson, M. (2006) Condition-dependent indicators in sexual selection: development of theory and tests. In Essays in Animal Behaviour: Celebrating 50 Years of Animal Behaviour (Lucas, J.R. and Simmons, L.W., eds), pp. 253-267, Elsevier

12 Fuller, R.C. et al. (2005) Sensory bias as an explanation for the evolution of mate preferences. Am. Nat. 166, 437-446

13 Kokko, H. et al. (2003) The evolution of mate choice and mating biases. Proc. R. Soc. B 270, 653-664

14 Jones, T.M. et al. (1998) Fisherian flies: benefits of female choice in a lekking sandfly. Proc. R. Soc. B 265, 1651-1657

15 Head, M.L. et al. (2005) The indirect benefits of mating with attractive males outweigh the direct costs. PLoS Biol. 3, 289-294

16 Tomkins, J.L. et al. (2004) Genic capture and resolving the lek paradox. Trends Ecol. Evol. 19, 323-332

17 Pomiankowski, A. and Iwasa, Y. (1998) Runaway ornament diversity caused by Fisherian sexual selection. Proc. Natl. Acad. Sci. U. S. A. 95, 5106-5111

18 Coyne, J.A. and Orr, H.A. (2004) Speciation, Sinauer

19 Erickson, D.L. et al. (2004) Quantitative trait locus analyses and the study of the evolutionary process. Mol. Ecol. 13, 2505-2522

20 Fitzpatrick, M.J. et al. (2005) Candidate genes for behavioural ecology. Trends Ecol. Evol. 20, 96-104

21 Thomas, M.A. and Klaper, R. (2004) Genomics for the ecological toolbox. Trends Ecol. Evol. 19, 439-445

22 Ryan, M.J. (1998) Sexual selection, receiver biases, and the evolution of sex differences. Science 281, 1999-2003

23 Rodd, F.H. et al. (2002) A possible non-sexual origin of mate preference: are male guppies mimicking fruit? Proc. $R$. Soc. $B$ 269, 475-481

24 Smith, C. et al. (2004) A receiver bias in the origin of three-spined stickleback mate choice. Proc. R. Soc. B 271, 949-955

25 Slate, J. (2005) Quantitative trait locus mapping in natural populations: progress, caveats and future directions. Mol. Ecol. 14, 363-379

26 Wayne, M.L. and McIntyre, L.M. (2002) Combining mapping and arraying: an approach to candidate gene identification. Proc. Natl. Acad. Sci. U. S. A. 99, 14903-14906

27 Barber, I. and Arnott, S.A. (2000) Split-clutch IVF: a technique to examine indirect fitness consequences of mate preference in sticklebacks. Behaviour 137, 1129-1140

28 Owens, I.P.F. (2006) Where is behavioural ecology going? Trends. Ecol. Evol. 21. doi: 10.1016/j.tree.2006.03.014

29 Gleason, J.M. et al. (2002) Quantitative trait loci affecting a courtship signal in Drosophila melanogaster. Heredity 89, 1-6

30 Civetta, A. and Cantor, E.J.F. (2003) The genetics of mating recognition between Drosophila simulans and D. sechellia. Genet. Res. 82, 117-126
31 Brooks, R. and Endler, J.A. (2001) Direct and indirect sexual selection and quantitative genetics of male traits in guppies (Poecilia reticulata). Evolution 55, 1002-1015

32 Evans, J.P. et al. (2003) Directional postcopulatory sexual selection revealed by artificial insemination. Nature $421,360-363$

33 Pilastro, A. et al. (2004) Cryptic female preference for colorful males in guppies. Evolution 58, 665-669

34 Houde, A.E. (1997) Sex, Color, and Mate Choice in Guppies, Princeton University Press

35 Magurran, A. (2005) Evolutionary Ecology: The Trinidadian Guppy, Oxford University Press

36 Williams, G.C. (1966) Adaptation and Natural Selection, Princeton University Press

37 Trivers, R.L. (1972) Parental investment and sexual selection. In Sexual Selection and the Descent of Man 1871-1971 (Campbell, B., ed.), pp. 136-179, Heinemann

38 Holland, B. and Rice, W.R. (1998) Chase-away sexual selection: antagonistic seduction versus resistance. Evolution 52,1-7

39 Gavrilets, S. et al. (2001) The evolution of female mate choice by sexual conflict. Proc. R. Soc. B 268, 531-539

40 Rice, W.J. et al. (2005) Inter-locus antagonistic coevolution as an engine of speciation: assessment with hemiclonal analysis. Proc. Natl. Acad. Sci. U. S. A. 102, 6527-6534

41 Cordero, C. and Eberhard, W.G. (2003) Female choice of sexually antagonistic male adaptations: a critical review of some current research. J. Evol. Biol. 16, 1-6

42 Parker, G.A. (1970) Sperm competition and its evolutionary consequences in the insects. Biol. Rev. 45, 525-567

43 Simmons, L.W. (2001) Sperm Competition and its Evolutionary Consequences in the Insects, Princeton University Press

44 Birkhead, T. and Møller, A.P. (1993) Female control of paternity. Trends Ecol. Evol. 8, 100-104

45 Eberhard, W.G. (1996) Female Control: Sexual Selection by Cryptic Female Choice, Princeton University Press

46 Bateman, A.J. (1948) Intrasexual selection in Drosophila. Heredity 2, 349-368

47 Simmons, L.W. (2005) The evolution of polyandry: sperm competition, sperm selection, and sperm viability. Annu. Rev. Ecol. Evol. Syst. 36, $125-146$

48 Keller, L. and Reeve, H.K. (1995) Why do females mate with multiple males? The sexually selected sperm hypothesis. Adv. Stud. Behav. 24, 291-315

49 Pizzari, T. and Birkhead, T.R. (2002) The sexually-selected sperm hypothesis: sex-biased inheritance and sexual antagonism. Biol. Rev. Camb. Philos. Soc. 77, 183-209

50 Eberhard, W.G. (1985) Sexual Selection and Animal Genitalia, Harvard University Press

51 Hosken, D.J. and Stockley, P. (2004) Sexual selection and genital evolution. Trend. Ecol. Evol. 19, 87-93

52 House, C.M. and Simmons, L.W. (2003) Genital morphology and fertilization success in the dung beetle Onthophagus taurus: an example of sexually selected male genitalia. Proc. R. Soc. B 270, $447-455$

53 Yasui, Y. (1997) A 'good-sperm' model can explain the evolution of costly multiple mating by females. Am. Nat. 149, 573-584

54 Kozielska, M. et al. (2004) Good genes and the maternal effects of polyandry on offspring reproductive success in the bulb mite. Proc. $R$. Soc. B 271, 165-176

55 Hosken, D.J. et al. (2003) Superior sperm competitors sire higherquality young. Proc. R. Soc. B 270, 1933-1938

56 Simmons, L.W. and Kotiaho, J.S. (2002) Evolution of ejaculates: patterns of phenotypic and genotypic variation and condition dependence in sperm competition traits. Evolution 56, 1622-1631

57 Møller, A.P. (1998) Sperm competition and sexual selection. In Sperm Competition and Sexual Selection (Birkhead, T.R. and Møller, A.P., eds), pp. 55-90, Academic Press

58 McLain, D.K. (1991) Components of variance in male lifetime copulatory and reproductive success in a seed bug. Behav. Ecol. Sociobiol. 29, 121-126

59 Westneat, D.F. and Stewart, I.R.K. (2003) Extra-pair paternity in birds: causes, correlates, and conflict. Annu. Rev. Ecol. Evol. Syst. 34, $365-396$ 
60 Safran, R.J. et al. (2005) Dynamic paternity allocation as a function of male plumage color in barn swallows. Science 309, 2210-2212

61 Bennett, P.M. and Owens, I.P.F. (2002) Evolutionary Ecology of Birds: Life Histories, Mating Systems and Extinction, Oxford University Press

62 Warner, R.R. et al. (1995) Sexual conflict: males with highest mating success convey the lowest fertilization benefits to females. Proc. $R$. Soc. $B$ 262, 135-139

63 Danielsson, I. (2000) Antagonistic pre- and post-copulatory sexual selection on male body size in a water strider (Gerris lacustris). Proc. R. Soc. B 268, 77-81

64 Preston, B.T. et al. (2001) Dominant rams lose out by sperm depletion. Nature 409, 681-682

65 Zeh, J.A. and Zeh, D.W. (1996) The evolution of polyandry I: intragenomic conflict and genetic incompatibility. Proc. R. Soc. B 263, 1711-1717

66 Tregenza, T. and Wedell, N. (2000) Genetic compatibility, mate choice and patterns of parentage. Mol. Ecol. 9, 1013-1027

67 Colegrave, N. et al. (2002) Mate choice or polyandry: reconciling genetic compatibility and good genes sexual selection. Evol. Ecol. Res. 4, 911-917

68 Mays, H.L. and Hill, G.E. (2004) Choosing mates: good genes versus genes that are a good fit. Trends Ecol. Evol. 19, 554-559

69 Stockley, P. (1997) Sexual conflict resulting from adaptations to sperm competition. Trends Ecol. Evol. 12, 154-159

70 Chapman, T. and Davies, S.J. (2004) Functions and analysis of the seminal fluid proteins of male Drosophila melanogaster fruit flies. Peptides 25, 1477-1490

71 Swanson, W.J. and Vacquier, V.D. (2002) The rapid evolution of reproductive proteins. Nat. Rev. Genet. 3, 137-144

72 Good, J.M. and Nachman, M.W. (2005) Rates of protein evolution are positively correlated with developmental timing of expression during mouse spermatogenesis. Mol. Biol. Evol. 22, 1044-1052

73 Orteiza, N. et al. (2005) Sexy sons from re-mating do not recoup the direct costs of harmful male interactions in the Drosophila melanogaster laboratory model system. J. Evol. Biol. 18, 1315-1323

74 Friberg, U. et al. (2005) Assessing the potential for an ongoing arms race within and between the sexes: selection and heritable variation. Evolution 59, 1540-1551

75 Byrne, P.G. and Rice, W.R. (2005) Remating in Drosophila melanogaster: an examination of the trading-up and intrinsic male-quality hypotheses. J. Evol. Biol. 18, 1324-1331

76 Møller, A.P. and Jennions, M.D. (2001) How important are direct fitness benefits of sexual selection? Naturwissenschaften 88, 401-415

77 Endler, J.A. and Basolo, A.L. (1998) Sensory ecology, receiver biases and sexual selection. Trends Ecol. Evol. 13, 415-420
78 Boughman, J.W. (2002) How sensory drive can promote speciation. Trends Ecol. Evol. 17, 571-577

79 Folstad, I. and Karter, A.J. (1992) Parasites, bright males, and the immunocompetence handicap. Am. Nat. 139, 603-622

80 Hamilton, W.D. and Zuk, M. (1982) Heritable true fitness and bright birds: a role for parasites? Science 218, 384-387

81 Iwasa, Y. et al. (1991) The evolution of costly mate preferences II. The 'handicap' principle. Evolution 45, 1431-1442

82 Rowe, L. and Houle, D. (1996) The lek paradox and the capture of genetic variance by condition dependent traits. Proc. $R$. Soc. B 263, 1415-1421

83 Houle, D. and Kondrashov, A.S. (2002) Coevolution of costly mate choice and condition-dependent display of good genes. Proc. R. Soc. B 269, 97-104

84 Neff, B. and Pitcher, T.E. (2005) Genetic quality and sexual selection: an integrated framework for good genes and compatible genes. Mol. Ecol. 14, 19-38

85 Bonduriansky, R. and Rowe, L. (2005) Sexual selection, genetic architecture, and the condition dependence of body shape in the sexually dimorphic fly Prochyliza xanthostoma (Piophilidae). Evolution 59, 138-151

86 Pizzari, T. and Birkhead, T.R. (2000) Female feral fowl eject sperm of subordinate males. Nature 405, 787-789

87 Wedell, N. et al. (2002) Sperm competition, male prudence and spermlimited females. Trends Ecol. Evol. 17, 313-320

88 Birkhead, T.R. (2000) Defining and demonstrating postcopulatory female choice - again. Evolution 54, 1057-1060

89 Calsbeek, R. and Sinervo, B. (2004) Within-clutch variation in offspring sex determined by differences in sire body size: cryptic mate choice in the wild. J. Evol. Biol. 17, 464-470

90 Olsson, M. et al. (1996) Sperm selection by females. Nature 383, 585

91 Marshall, R.C. et al. (2003) Sexual selection and individual genetic diversity in a songbird. Proc. $R$. Soc. B 270, S248-S250

92 Folstad, I. and Skarstein, F. (1997) Is male germ line control creating avenues for female choice? Behav. Ecol. 8, 109-112

93 Rolff, J. (2002) Bateman's principle and immunity. Proc. R. Soc. B 269, $867-872$

94 Skau, P.A. and Folstad, I. (2005) Does immunity regulate ejaculate quality and fertility in humans? Behav. Ecol. 16, 410-416

95 Jacot, A. et al. (2004) Costs of an induced immune response on sexual display and longevity in field crickets. Evolution 58, 2280-2286

96 Faivre, B. et al. (2003) Immune activation rapidly mirrored in a secondary sexual trait. Science 300,103

97 Blount, J.D. et al. (2003) Carotenoid modulation of immune function and sexual attractiveness in zebra finches. Science 300, 125-127

98 Simmons, L.W. and Roberts, B. (2005) Bacterial immunity traded for sperm viability in male crickets. Science 309, 2031

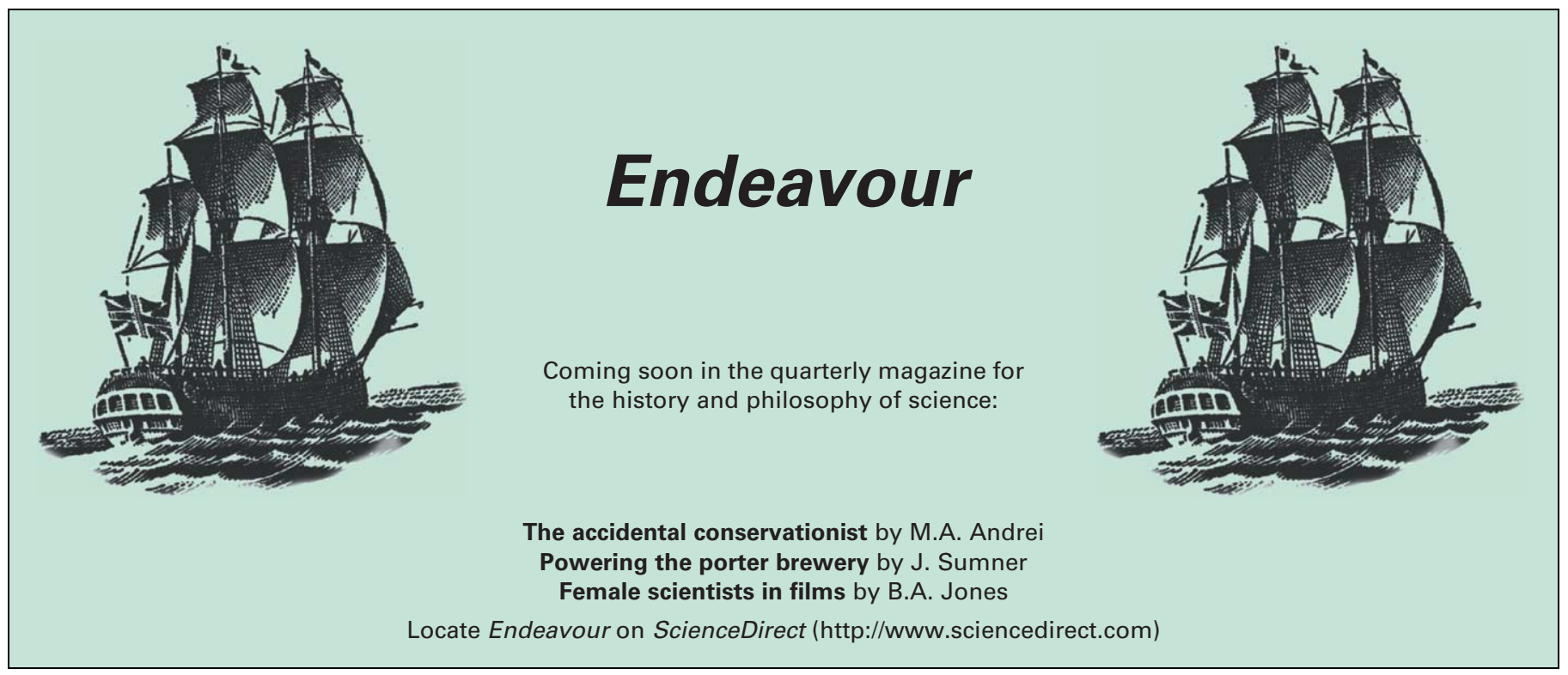

